\title{
Research on path planning of multi picking robot based on ant colony algorithm
}

\author{
Lingmin Liu, Jing Hu, Qian Xie \\ Computer School, Wuhan Qingchuan University,Wuhan,430204,China
}

Keywords: ant colony algorithm; picking robot; path planning; navigation

\begin{abstract}
With the rapid development of computer technology, information technology, artificial intelligence theory and sensor technology, the robot research has reached a new height. In order to solve the problem of path conflict and collision among multi robot groups, based on ant colony algorithm, the design and research of multi picking robot path planning and navigation system, realizes the multi mobile robot real-time dynamic picking path planning and autonomous navigation function in this paper, which can provides a theoretical basis for the further development of robot.
\end{abstract}

\section{Introduction}

In recent years, artificial intelligence has become the cornerstone of the new round of industrial revolution. It is not only widely used in industrial control field, but also more and more popular in agricultural production. In the busy season, there will be a large number of farms tend to labor shortage phenomenon, therefore, the robot in agricultural applications demand more and more obvious. The development of a remote control of the picking robot, instead of the traditional human operation, has important significance to improve the level of agricultural production, improve the efficiency of planting, but also the development trend of modern agriculture. According to the working environment of picking robot, ant colony algorithm is used to design and study a path planning and navigation system for multiple harvesting robots.

\section{Brief introduction of ant colony algorithm}

Ant colony algorithm is a method of solving complex combinatorial optimization problems, which was simulated and summarized by Italy scholar Dorigo in 1990s. Talk about the principle, we must search for food from the behavior of ants and bees of these insects, in fact, their behavior is very easy to analyze for an individual, but when they are combined into a large group, the group behavior will greatly increase the difficulty of analysis. The reason is because the individual ant is a kind of secretions as pheromone communication, ants will leave these substances in walking routes, and all the ants are able to perceive the concentration of these substances, and according to the concentration of a substance to their choice of motion intensity. Therefore, the collective behavior of the ant will form a positive feedback information specific to the scene, after a number of ants on the route is bigger, more ants will choose behind the route, ants according to the perception of this pheromone substances can implement the whole process of the search for food, this is also the reason of the ant colony algorithm. The ants look for the food process as shown in Figure 1. 


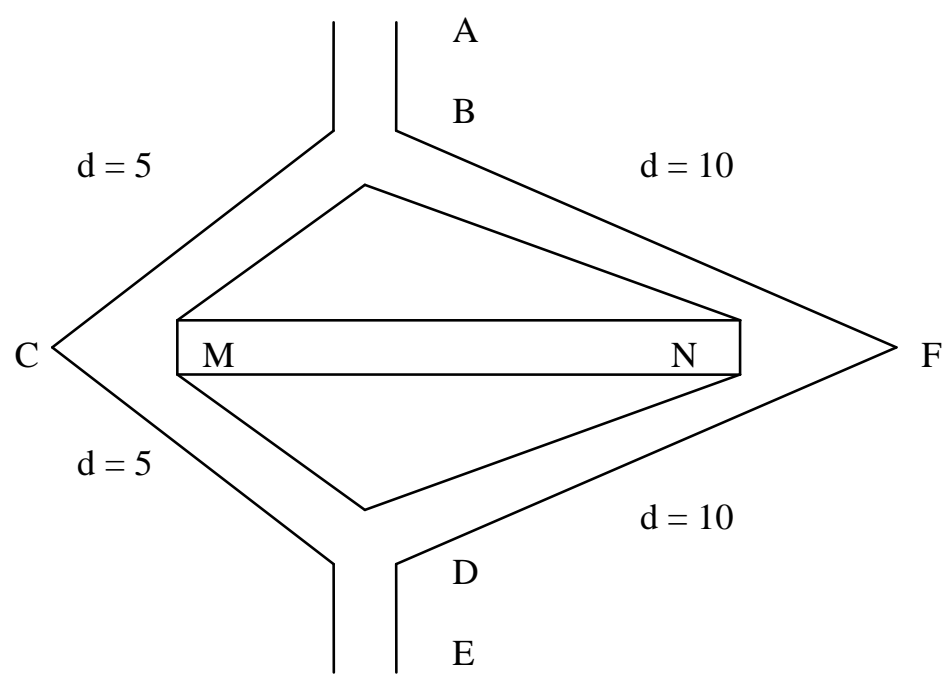

Fig. 1 Schematic diagram of ant colony searching for food process

As shown in Figure 1, assuming that $\mathrm{A}$ is the ant colony location, $\mathrm{E}$ is the place where there is food, and MN is a barrier between A and E. Since there is an obstacle MN between A and E, then the ant can only move the food from two paths of BCD and BFD, of which the length of the two paths are 10 and 20, respectively. Suppose that there are 200 ants moving from A to E in one minute, and the total number of pheromones increases by 20 after the ants pass. In order to make the calculation simpler, the pheromone is generated from the time of generation to the disappearance of 10 minutes. In the beginning, no pheromone material existence, probability of ants select the two lines of the same, but because the BCD path is short, ants choose less, the time required by the BCD and, at the same time, through the BCD of the ants will be more, so the BCD path pheromone material more than the BFD. It will attract the ants behind from BCD, so as to realize the optimal path from A to E.

\section{Path planning for multiple picking robots}

In the process of multi picking robot, there are many obstacles, the robot affects each other, and they become obstacles to each other. Therefore, the path planning of multi robot is difficult. In actual research, many harvesting robots work in the same planting area, and many optimal paths need to be planned, but in the specific operation process, due to the presence of obstacles, the optimal path will change, it will also affect the other path of mobile robot. Based on the above problems, in order to ensure the collision free operation of the multi picking robot, the system needs to plan the route in real-time to prevent collisions between the robot, the robot and the obstacle.

Because of the Industrial Park planting environment uncertainty factors, such as planting Park work and random moving machinery, the sensor has some limitations, when the robot is in the initial planning is often difficult to obtain all the information, therefore, the optimal path planning is often difficult to obtain simple ideal. Therefore, on the basis of getting the environmental information of a certain Park, we can make the global planning before the job and take it as the ideal optimal route. In the actual operation picking machine in a population, according to the subsequent detection of environmental information, the robot must plan the optimal path of the local, simple fine-tuning, make the robot avoid all collision, and reach the destination as fast as possible. Based on the above problems, the global and local planning method is adopted to realize the path planning of the multi picking robot. The path planning framework of a multi picking robot is shown in Figure 2. 


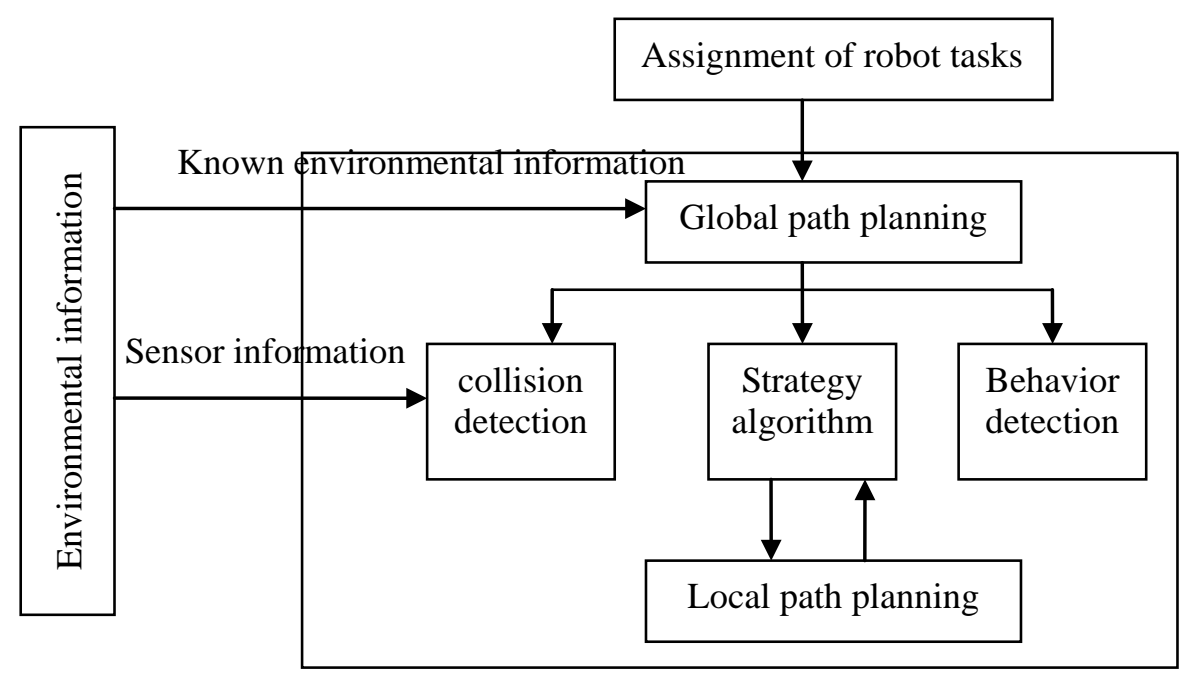

Fig.2 Path planning framework

\section{Global path planning}

This chapter is the study of the path planning algorithm, multi picking robot in the actual operation process of the park, for its global path planning, the first thing to do is in accordance with the requirements of the specific operation, according to the known information, assign tasks to each robot, and determine the starting point of each machine operation and end point, and then combined with the cooperation the mechanism of multi robot, planning a collision free optimization route between two points. In the whole algorithm, the most important is to improve the route planning speed and realize the optimality of path planning. Because ant algorithm is more efficient in path planning, and it has stronger adaptability to obstacle change, ant colony algorithm will be used for global path planning of multi picking robot.

\section{Local path planning}

With the global path planning, picking the machine crowd can start their own tasks. Initially, picking robot path planning will be in accordance with the respective operations, and collect the information of surrounding environment, according to the dynamic information collected, analyzed, modify the local path according to collision detection, decision algorithm and behavior detection, which itself has stronger ability to adapt to the environment.

Local planning algorithm in this system is used for the real-time detection of the surrounding environment information by sensors on the robot, and the results were compared with the global planning information, according to the collision detection system to assess the risk of collision exists. If there is a danger of collision, the local path must be adjusted. In the process of adjustment, the end of the job is always taken as the final goal, and the local extreme point is avoided, while the whole planning of the robot is neglected. In addition, in the local planning process, the behavior detection subsystem needs to determine the path of the picking robot in real time and determine whether it meets the goal of global planning. After the robot successfully avoids the obstacle, it can continue the job operation according to the global planned route, and move to the nearest sub path node during the execution of the desired path. Again and again, no matter how the local planning path is implemented, the final destination of the picking robot will not change until it reaches the finish line successfully.

\section{Multi picking robot navigation system}

Integrated navigation system program. Multi picking robot navigation system is that during the execution of the task, the robot navigation operation line, according to the overall planning of the combination of the information environment in the forward process is perceived, real-time appropriate modifications to the route, leading the robot to the specified location. The scheme mainly includes three parts: environment modeling, autonomous localization and path planning. 
Because path planning has been elaborated in the previous chapter, it is no longer repeated here.

(1) Environment modeling

Environment modeling refers to the method of mathematical model, which describes the picking area in detail, and abstracts it into the data information which can be directly identified by the computer system. In this paper, the navigation system data information is in the background server, and the topological graph is abstracted by this information, and the route information is simply described. In addition, it is convenient for each picking robot to identify the links between the roads and realize global, local path planning and real-time navigation.

(2) Picking robot localization

Picking robot localization is based on the surrounding environment information and GPS to determine its location in the work area relative to the global coordinates.

Concrete implementation of environmental modeling. The spatial information of environment modeling is crucial to realize the navigation of multi picking robot, the method used in this paper is to map information in the background server, draw the topology map through the database of the back-end server, and save the data information by the way of circular linked list. Therefore, the backstage database in the server to store all information map, can be used for real-time positioning and navigation of multi robot, and can be used in combination with the topology of ant algorithm to realize optimal path planning.

(1) Map information database

The map information is divided into the station and two Station Road, the area is relatively large, large, and it can be used to make robots for short stay; and the road is the route for the robot to move forward, to connect the station.

Second, in the planning of the strategy algorithm, the station and the road can be regarded as a section or a block of matrices, and can be connected to represent the map. The map information is shown in figure 3.

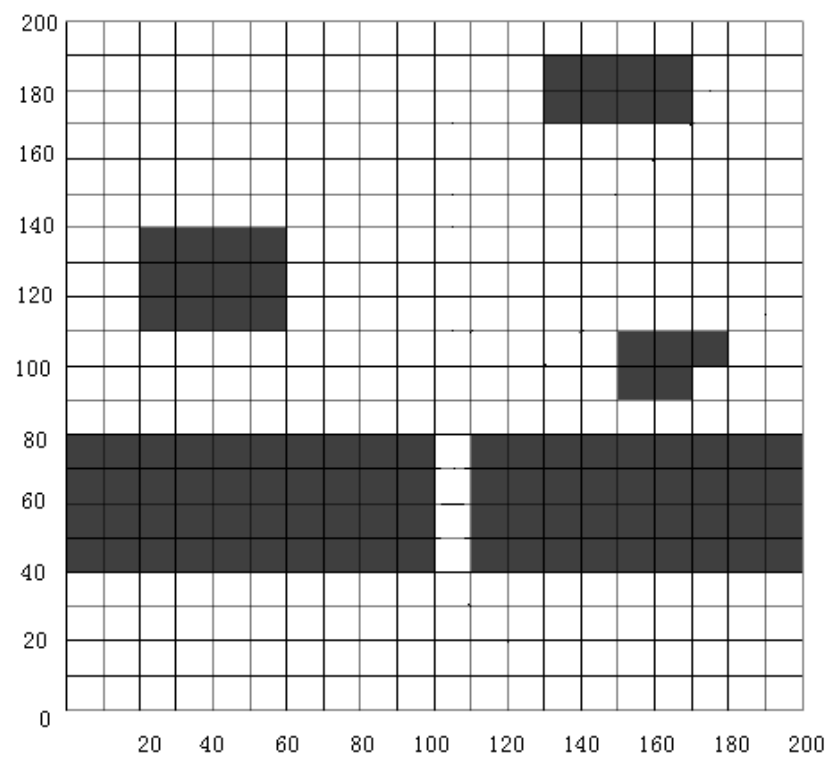

(2) Topology diagram

Fig. 3 Build navigation map information diagram

The establishment of the topology needs to be based on the data information in the server, which is mainly used to describe the connection status between the sites and the road, and store them in memory by means of circular linked lists. The navigation map topology is shown in Figure 4. 


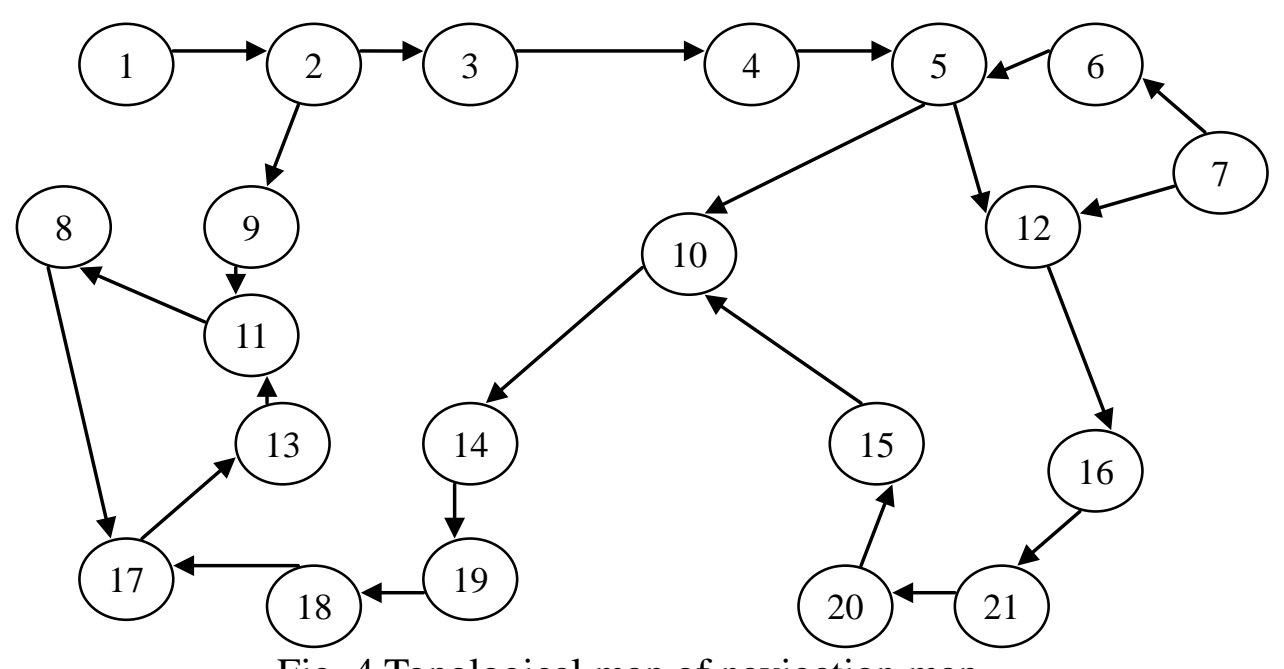

Fig. 4 Topological map of navigation map

As shown in Figure 4, the circular list is a method commonly used to save the data information in the data structure, which can flexibly represent data and see, find one of the information, we can know the memory inside information. In the process of navigation, the connection information between nodes is often accessed, so this method can greatly improve the data reading information.

\section{Conclusion}

In order to solve the problem of path conflict and collision among multi robot groups, multi picking robot path planning and navigation system has been designed and researched based on ant colony algorithm in the paper. Due to the Industrial Park planting environment uncertainty factors, and the robot is often difficult to obtain all the information when the initial planning, the global and local path planning method is proposed to realize the real-time dynamic programming of the mobile path of a multi picking robot. In picking robot environment, method of autonomous navigation based on environment modeling and localization of the robot is put forward, through the establishment of a map information database and topology, the safety guide of picking robot has been realized.

\section{Acknowledgements}

The work was supported by the guidance project of science and technology research plan of Hubei Provincial Department of Education with the project number B2016419 and the project name Research on surveillance video monitoring system based on mobile Internet.

\section{References}

[1] Zhang Wei. Study on the construction of map of indoor mobile robot based on binocular stereo vision [D]. Wuhan University of Science and Technology. (2009).

[2] Tang Huikang, Zheng Baolin. Research on remote formation control of picking robot based on SingalR and [J]. Journal of Agricultural Mechanization Research, No.03, (2017), p.237-241.

[3] Ge Sinan. Multi robot task allocation based on improved ant colony algorithm[D]. Shenyang University of Technology, (2016)

[4] Wang Youfa. Research on task allocation for multi robot systems oriented to intelligent manufacturing [D]. Nanjing University, (2016).

[5] Wang Wei. Research and application of multi robot path planning method based on multi-agent system [D]. Electronic Science and Technology University, (2015).

[6] Wang Li. Research on path planning method of mobile robot [D]. Northwestern Polytechnical University, (2007). 
[7] Li Jing. Research on path planning, collision avoidance and coordination of multi mobile robots [D]. Shanghai Jiao Tong University, (2007).

[8] Lv Qiang. Research on information perception technology and path planning for working scene of Citrus picking robot [D]. Jiangsu University, (2010).

[9] Li Rui, Han Yongxiang. An improved ant colony algorithm in distribution send on the [J]. Computer Knowledge and Technology, Vol. 22, (2009), p.6290-6291.

[10] Shao Jie, Yang Jingyu, Du Lijuan. A hybrid hierarchical architecture for multi robot based on improved learning classifier [J]. Journal of Nanyang Institute of Technology, No.03, (2009), p.21-24.

[11] Liu Xiaomei, Zhang Junjing. Basic principle and application of ant colony optimization algorithm [J]. West-China Exploration Engineering, No.10, (2008) , p.240-243.

[12] He Wu. Research on Routing Algorithms for wireless sensor networks based on ant colony optimization algorithm [D]. Southwest Jiao Tong University, (2008). 